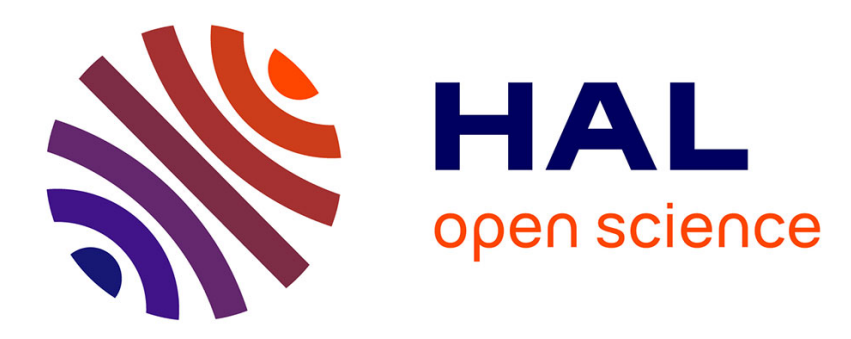

\title{
The Development of Organic Conductors: Organic Superconductors
}

\author{
Denis Jerome
}

\section{To cite this version:}

Denis Jerome. The Development of Organic Conductors: Organic Superconductors. 2008. hal00255879

\section{HAL Id: hal-00255879 \\ https://hal.science/hal-00255879}

Preprint submitted on 26 Feb 2008

HAL is a multi-disciplinary open access archive for the deposit and dissemination of scientific research documents, whether they are published or not. The documents may come from teaching and research institutions in France or abroad, or from public or private research centers.
L'archive ouverte pluridisciplinaire HAL, est destinée au dépôt et à la diffusion de documents scientifiques de niveau recherche, publiés ou non, émanant des établissements d'enseignement et de recherche français ou étrangers, des laboratoires publics ou privés. 


\title{
The Development of Organic Conductors: Organic Superconductors
}

\author{
Denis Jérome \\ Université Paris-Sud,91405, Orsay, France
}

February 14, 2008

This article provides a brief account of the development of organic conductors since the seventies. This research has been motivated by the quest for high $T_{c}$ superconductors. Besides superconductivity found in organics in 1980, progresses in this field of research have highlighted the wealth of remarkable properties of low dimensional physics.

\section{Searching for high $T_{c}$ superconductors}

Superconductivity ( a new state of matter) has been one of the major discovery of the twentieth century in physics. From an experimental point of view the finding by Kamerlingh Onnes[1] in 1911 of a current travelling without resistance through a metal cooled at very low temperature paved the way to a very large number of industrial applications. And this, long before the proposal of a satisfactory theoretical framework by Bardeen, Cooper and Schrieffer (BCS)[2] in 1957 explaining both the zero resistance state and the magnetic flux expulsion ( the Meissner effect). Furthermore, the BCS theory has also been the paradigm of modern physical theories based on the importance of the quantum nature at low temperature since it relies on the establishment of a new long range ordered state. BCS emphasized the existence of a two body attractive interaction between charge carriers (either electrons or holes) being a prerequisite for a Bose condensation of electron pairs into the superconducting state. This net attractive coupling in spite of the Coulomb repulsion between carriers of the same sign relies closely on an attraction between electrons mediated by their interaction with excitations of the lattice namely, the phonons.

Consequently, a major achievement of the BCS theory has been the understanding of the ionic mass dependent of the critical temperature i.e. $\left(T_{c} \alpha M^{-1 / 2}\right)$. Although Fröhlich had proposed earlier in 1954 a model for superconductivity based on the involvement of the lattice [3] this theory were unable to account for the superconductivity of metals but turned out to be quite relevant later for the interpretation of the transport properties in some one dimensional organic compounds, vide infra. At the beginning of the 60's searching for new materials exhibiting the highest possible values of superconducting tc was already 


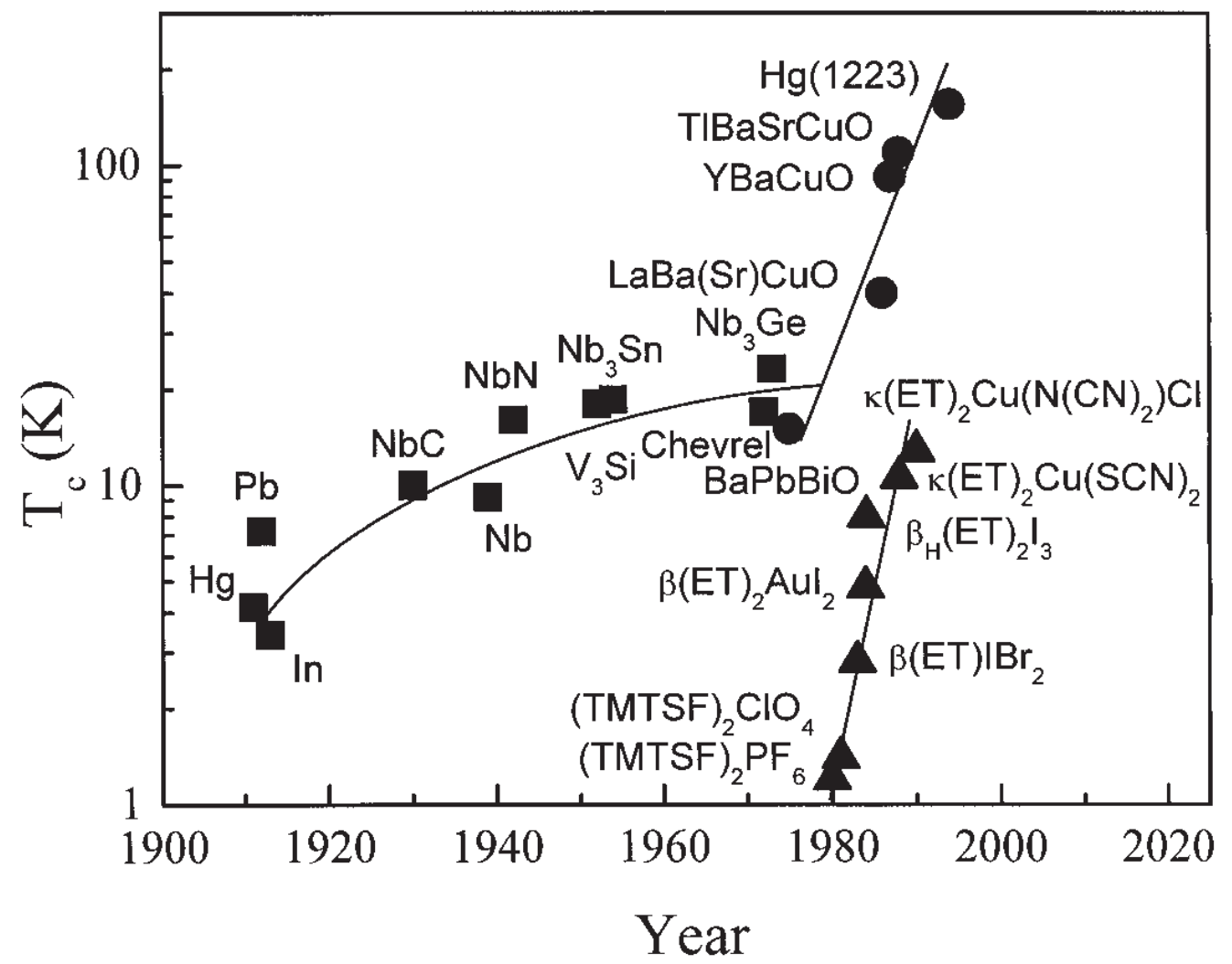

Figure 1: Evolution of the superconducting critical temperature in metals and intermetallic compounds, cuprates and organic conductors (1 and 2D conductors) . 
a strong motivation in materials sciences and the term high temperature superconductor was already commonly used refering to the intermetallic compounds of the A15 structure, namely, $\left(\mathrm{Nb}_{3} \mathrm{Sn}\right.$ or $\left.\mathrm{V}_{3} \mathrm{Si}\right)[4]$. The hidden 1D nature of the A15 structure provides an enhancement of the density of states at the Fermi level lying close to the van-Hove singularity of the density of states of the $1 \mathrm{D}$ d-band. Within the BCS formalism large $T_{c}$ could be expected. They were actually observed (17-23K) but an upper limit was found to the increase of $T_{c}$ since the large value of $\mathrm{N}\left(\mathrm{E}_{\mathrm{F}}\right)$ makes also the structure unstable against a cubic to tetragonal band Jahn-Teller distortion $[5,6]$. The theory showed that the actual compounds $\mathrm{Nb}_{3} \mathrm{Sn}$ and $\mathrm{V}_{3} \mathrm{Si}$ possess their optimum $T_{c}$ [7]. In this context some papers at the beginning of the 80 's, based on metallurgical considerations, regarded $25-30 \mathrm{~K}$ as the highest possible value for $T_{c}[8]$. However these attempts to increase $T_{c}$ were still based on the BCS theory and its strong coupling extension $[9,10]$. Expending the successful idea of the isotope effect in the BCS theory other models were proposed in which excitations of the lattice responsible for the electron pairing had been replaced by higher energy excitations namely, electronic excitations, with the hope of new materials with $T_{c}$ higher than those explained by the BCS theory. Consequently, the small electronic mass $m_{e}$ of the polarizable medium would lead to an enhancement of $T_{c}$ of the order of $\left(M / m_{e}\right)^{1 / 2}$ times the value which is observed in a conventional superconductor, admittedly a huge factor. V.L.Ginzburg[11, 12] considered in 1964 the possibility for the paring of electrons in metal layers sandwiched between polarizable dielectrics through virtual excitations at high energy. W.A.Little made an important suggestion[13, 14]: a new mechanism for superconductivity expected to lead to a drastic enhancement of the superconducting $T_{c}$ to be observed in especially designed macromolecules. The idea of Little was indeed strongly rooted in the extension of the isotope effect proposed by BCS. However a prerequisite to the model of Little was the achievement of conduction in molecular crystals namely, new types of conductors in which the conduction would proceed through the transport of charge between molecular orbitals rather than atomic orbitals. Actually, the concept of a synthetic metal had aleady been launched by McCoy and Moore[15] when they proposed to prepare composite metallic substances from non-metallic constituent elements. As to the possibility of superconductivity in materials other than metals, Fritz London in 1937[16] has been the first to suggest that compounds with aromatic rings such as anthracene, naphtalene,... might exhibit a current running freely around the rings under a magnetic field. The first successful attempt to promote metal-like conduction between open shell molecular species came out in 1954 with the synthesis of the molecular salt of perylene oxidized with bromine [17] although this salt was rather unstable. Coming back to 1D materials, the model of Little was based on the use of a long conjugated polymer such as a polyacetylene molecule grafted by polarizable side groups[18] (see figure 2). Admittedly, this formidable task in synthetic chemistry has not reached its initial goal but the idea to link organic metallicity and one dimensionality was launched and turned out to be a very strong stimulant for the development of organic superconductors. Needless to say that a lot of basic physical problems had been overlooked in the seminal paper of Little[13] which was still based on 
the popular Fermi liquid BCS theory. The first point is that one dimensional conductors had been regarded previously as textbook examples by Peierls[19], who stated a theorem according to which a gap should spontaneously open at the Fermi level in the conduction and lead to the stabilization of a dielectric insulating state at low temperature long before it has been established in the platino-cyanate chains[20]. Bychkov et-al[21] criticized the model of Little regarding its potentiality to lead to high temperature superconductivity. Following Bychkov et-al, the one-dimensional character of the model system proposed by Little makes it a unique problem in which there exists a built-in coupling between superconducting and dielectric instabilities. It follows that each of these instabilities cannot be considered separately in the mechanism proposed by Little in one dimension and last but not least, fluctuations should be very efficient in a $1 \mathrm{D}$ conductor to suppress any long range ordering to very low temperature. A major step has been accomplished in 1970 towards the discovery of new materials for superconductivity with the synthesis by F.Wudl[22] of the new molecule tetrathiafulvalene, (TTF ). This molecule containing four sulfur heteroatoms in the fulvalene skeleton can easily donate electrons when it is combined to electron accepting species. This discovery has deeply influenced the subsequent evolution of the chemistry of organic conductors.

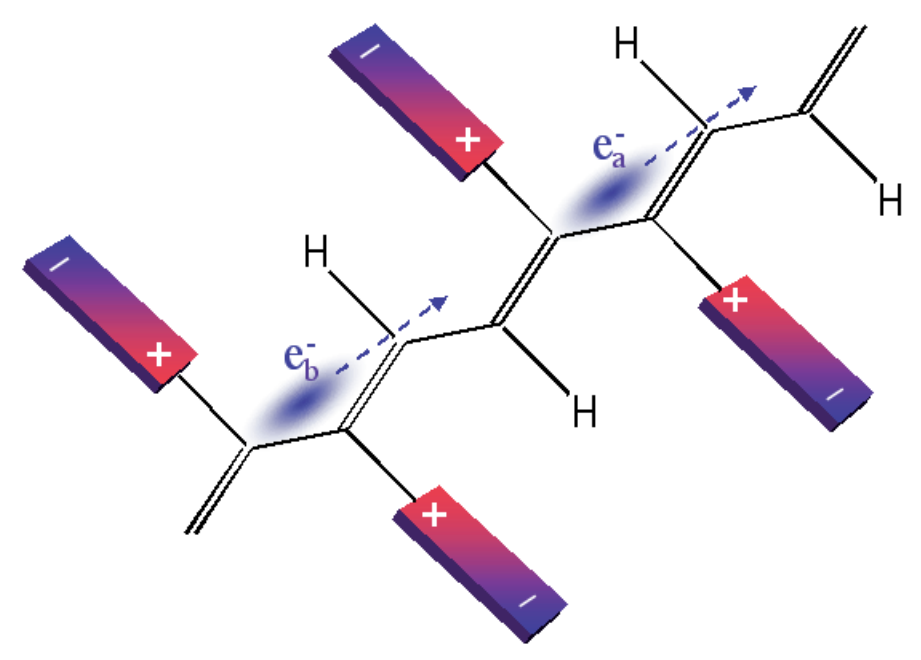

Figure 2: Schematic picture of Little's suggestion. Charge carriers $a$ and $b$ moving along a conducting spine are bound via a virtual electronic excitation of polarizable side groups.

\section{First organic conductors: TTF-TCNQ}

The discovery of the molecule TTF triggered the synthesis of the first stable organic metal, a charge transfer complex, TTF - TCNQ. The system is made up of two kinds of flat 
molecules each forming segregated parallel conducting stacks. This compound can be recognized as an organic conductor as the orbitals involved in the conduction $(\pi$-HOMO and $\pi$-LUMO for TTF and TCNQ respectively) are associated with the molecule as a whole rather than with a particular atom with carriers in each stacks provided by an interstack charge transfer at variance with other organic conductors such as the doped conjugated polymers which came out a little bit later. The overlap of the molecular orbitals being largest along the stack direction and much weaker between them makes the electron dispersion one dimensional (1D). In a first approximation the energy of this 1D conductor depends only on the electron wave vector along the $b^{*}$ direction in the reciprocal space and due to the inverted band structure coming from TTF and TCNQ respectively the 1D bands have to intersect the Fermi energy at Fermi wave-vectors $\pm k_{F}$. In turn, the band filling is not determined by stoechiometric considerations but rather by a subtle chemical balance. Hence, the band filling does not need to be commensurate with the underlying lattice.

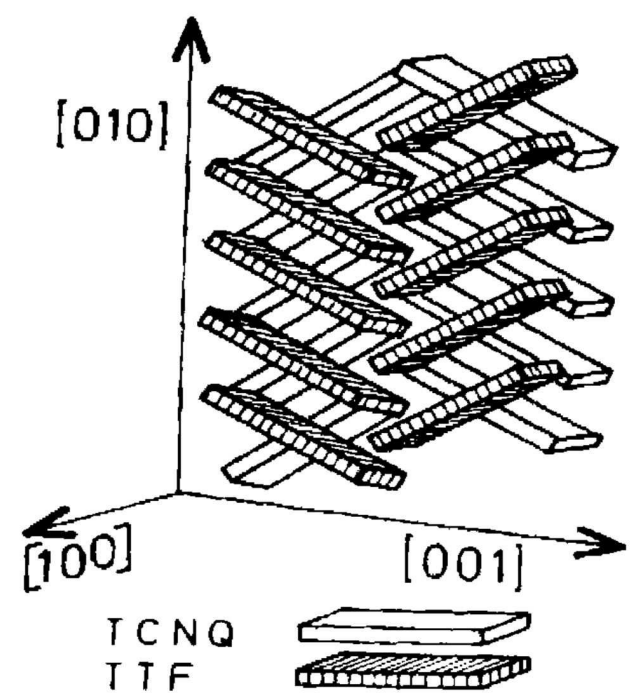

Figure 3: An artist view of the TTF - TCNQ stacked structure according to reference[23].

The announcement of a large and metal-like conduction in TTF - TCNQ was made in 1973, simultaneously by the Baltimore[24] and Pennsylvania[25] groups. The Pennsylvania group made a stimulating claim announcing a giant conductivity peak of the order of $10^{5}(\Omega \mathrm{cm})^{-1}$ at $60 \mathrm{~K}$ arising just above a very sharp transition towards an insulating ground state at low temperature. This conductivity peak was attributed by their authors to precursor signs of an incipient superconductor. The claims for superconductivity were made on the basis of $1 \mathrm{D}$ superconducting fluctuations enhanced by the softening of the $2 k_{F}$ phonon mode coupled to the electrons. This was still traditional thinking since it was believed from the study of the A15 superconductors that soft phonons modes were 
responsible for the high values of their $T_{c}$ [8]. Unfortunately,a conductivity peak with such a giant amplitude could never be reproduced by other groups who anyway all agreed on the metallic character of this novel molecular material[26]. Quite important has been the

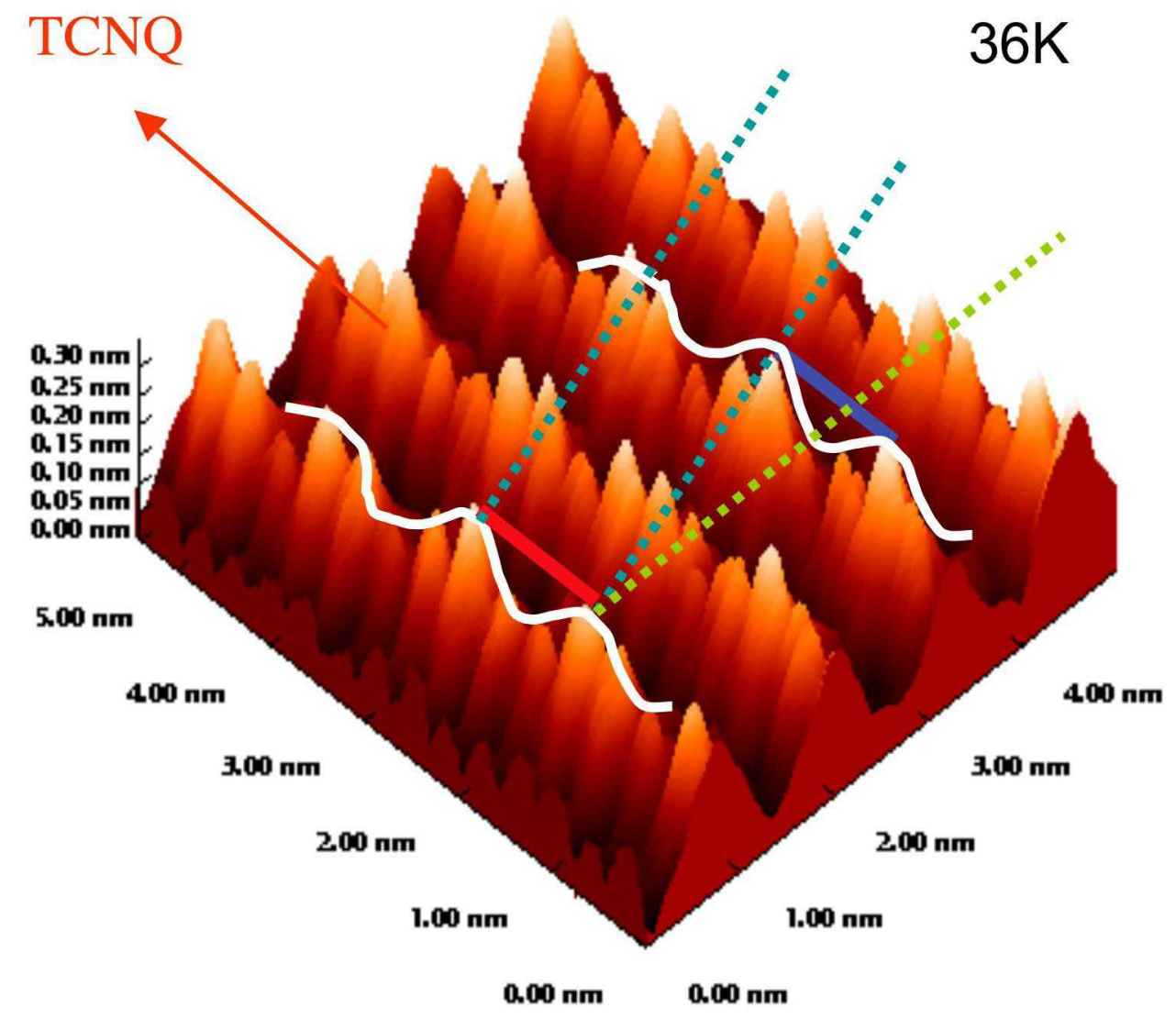

Figure 4: STM data from reference[27] showing the charge density wave at $36 \mathrm{~K}$ on TCNQ stacks with longitudinal $\lambda_{b}=3.39 b$ and commensurate transverse ordering , $\lambda_{a}=4 a$.

finding by the Orsay group from X-ray scattering studies of Bragg satellites allowing to link the metal-insulator transition to the instability predicted by Peierls[28] in a conducting chain. The Peierls transition leading to a charge density wave structure has been recently reexamined in the direct space by STM techniques[27], (see, figure 4).

High pressure transport measurements have played for the first time a decisive role in the study of organics as shown by the study of the temperature-pressure phase diagram of TTF - TCNQ. As displayed on fig. 5, the sharp peak of the Peierls transition 
superimposed to a slow increase under pressure has been attributed to a third order commensurability pinning, the wave length of the Peierls distorsion become commensurate with the underlying lattice in the vicinity of $19 \mathrm{kbar}\left(\lambda_{\|}=3 b\right)$ also confirmed by direct neutron scattering data[29]. Taking advantage of a commensurability obtainable under
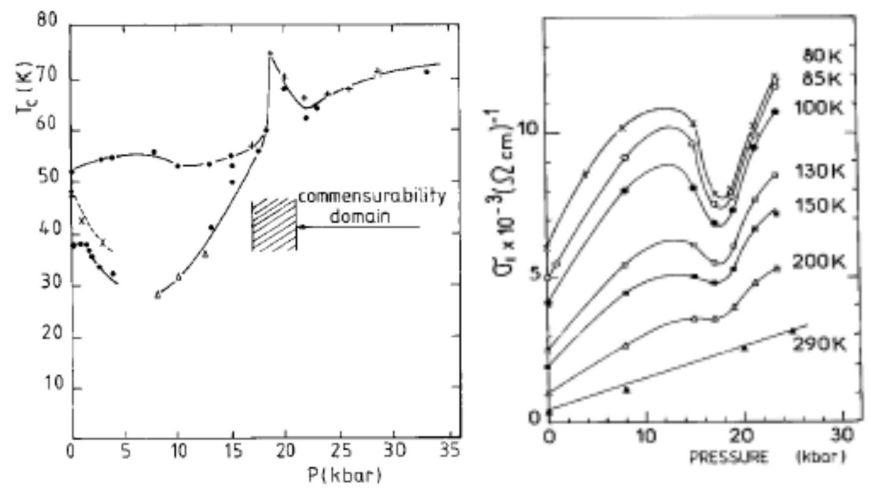

Figure 5: Temperature-pressure phase diagram of TTF - TCNQ showing the commensurability domain around 14-19kbar (left)[30] and longitudinal conduction measured versus pressue under constant temperature displyaing a drop of the conduction in the commensurability (x3) domain due to pinning of the fluctuating Fröhlich contribution.

pressure the possibility to perform systematic studies of the conductivity in the metallic temperature domain by sweeping the helium gas pressure at fixed temperatures did show a drop of longitudinal conductivity closely related to the commensurability conditions[31]. This behaviour brought the experimental confirmation of collective Fröhlich fluctuations in a $1 \mathrm{D}$ regime above a Peierls transition and, to a large extent, contributed to the understanding of the conduction peak. Furthermore, the role of 1D electron-electron repulsive interactions has been recognized for the first time in organics by the $4 \mathrm{k}_{F}$ signature in X-ray diffuse scattering experiments[32].

While high pressure appeared to be a parameter far more influential for organic conductors than for regular metals, it has failed until recently[33] to make TTF - TCNQ sufficiently three dimensional to prevent the onset of a Peierls instability and enable the stabilisation of superconductivity[34].

\section{Superconductivity in the Bechgaard salts}

While the study of TTF - TCNQ was going on it was recognized that electron polarizability is an important factor to reduce the screened on-site e-e repulsion. Therefore, the redox potential $(\Delta E)_{1 / 2}$ should be minimized, $[35,36]$ in order to fulfill this goal and obtain potentially conducting materials. Hence, new charge transfer compounds with the acceptor 
TCNQ were synthesized using other heteroatoms besidfe sulfur for the donor molecule, i.e. substituting sulfur for selenium in the TTF skeleton thus leading to the TSeF molecule. Much attention was put on the tetramethylated derivative of the TSeF molecule which, when combined to the dimethylated TCNQ gave rise to TMTSF - DMTCNQ . The outcome of this study has been truly decisive for the quest of organic superconductivity [37] since for the first time a metallic state could be stabilized under pressure in an organic compound as a ground state instead of the usual Peierls ground state observed in all the other compounds belonging to the TTF - TCNQ series[38]. In 1979, the Montpellier group synthesized a series of isomorphous radical cationic conductors based on TMTTF (the sulfur analog of the TMTSF molecule) with an inorganic anion[39]. These materials were all insulating at ambient pressure but some of them did show superconductivity under pressure 20 years later and became quite important for the physics of $1 \mathrm{D}$ conductors. At the

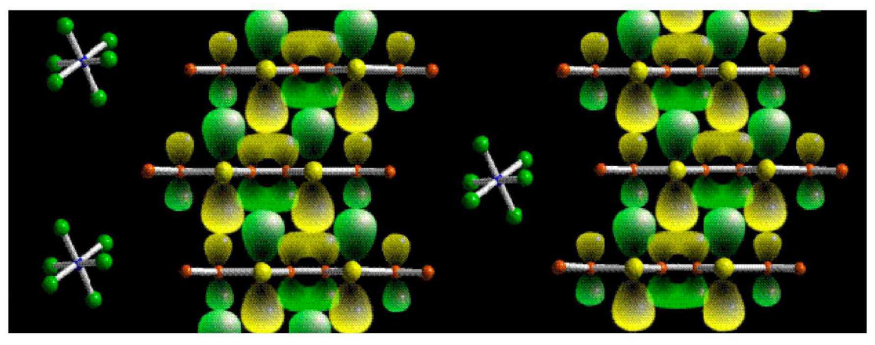

Figure 6: Side view of $(\mathrm{TM})_{2} \mathrm{X}$ conductors along the $b$ axis.

same time, the Copenhagen group led by Klaus Bechgaard succeeded in the synthesis of a new series of conducting salts all based on the TMTSF molecule namely, (TMTSF) ${ }_{2} \mathrm{X}$ where $\mathrm{X}$ is an inorganic mono-anion with various possible symetry, spherical $\left(\mathrm{PF}_{6}, \mathrm{AsF}_{6}\right.$, $\left.\mathrm{SbF}_{6}, \mathrm{TaF}_{6}\right)$, tetrahedral $\left(\mathrm{BF}_{4}, \mathrm{ClO}_{4}, \mathrm{ReO}_{4}\right)$ or triangular $\left(\mathrm{NO}_{3}\right)[40]$. All these compounds but the one with $\mathrm{X}=\mathrm{ClO}_{4}$ did reveal an insulating ground state under ambient pressure. What is special with (TMTSF) ${ }_{2} \mathrm{PF}_{6}$, the prototype of the so-called Bechgaard salt, unlike previously investigated TTF - TCNQ, is the magnetic origin of the ambient pressure insulating state related to the onset of itinerant antiferromagnetism opening a gap at Fermi level over the planar (1D) Fermi surface.

\section{Beyond superconductivity: the generic $\mathrm{TM}_{2} \mathrm{X}$ diagram}

In the mid-1980's, the isostructural family comprising the sulfur molecule with the same series of monoanions was investigated under pressure and it was realized that (TMTTF) ${ }_{2} \mathrm{X}$ and (TMTSF $)_{2} \mathrm{X}$ salts both belong to a unique class of materials forming the generic $(\mathrm{TM})_{2} \mathrm{X}$ phase diagram[42], figure.(8). In particular, (TMTTF $)_{2} \mathrm{PF}_{6}$, although the most insulating compound of the phase diagram can be made superconducting at low temperatures under a pressure of $45 \mathrm{kbar}[43,44]$. Because of their particular crystal structures, such materials 


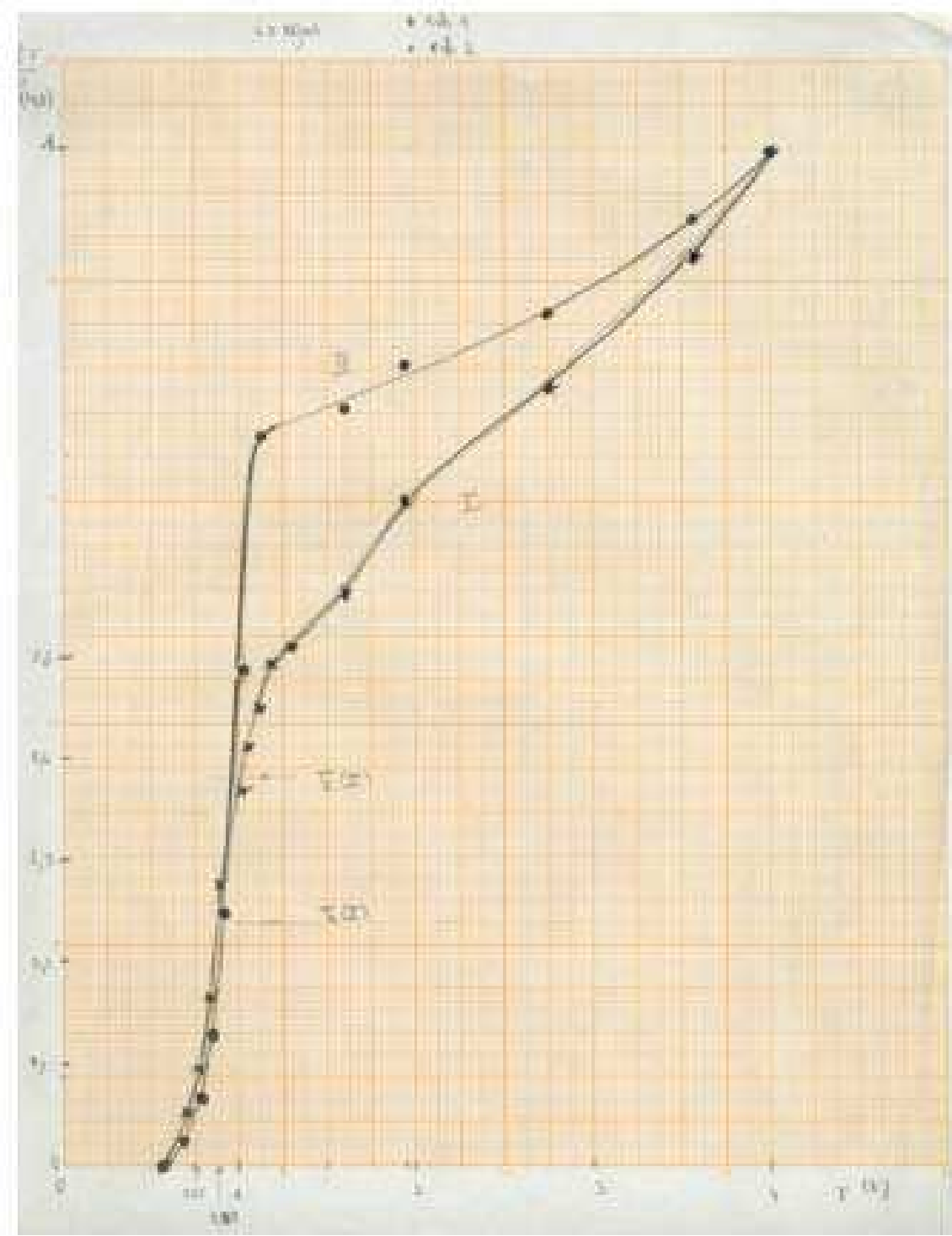

Figure 7: The first observation of organic superconductivity in (TMTSF) ${ }_{2} \mathrm{PF}_{6}$ under pressure[41].The resistance of two samples is displayed. This is a fac-simile of the 1979 lab book, before the common use of computerized data acquisition. 


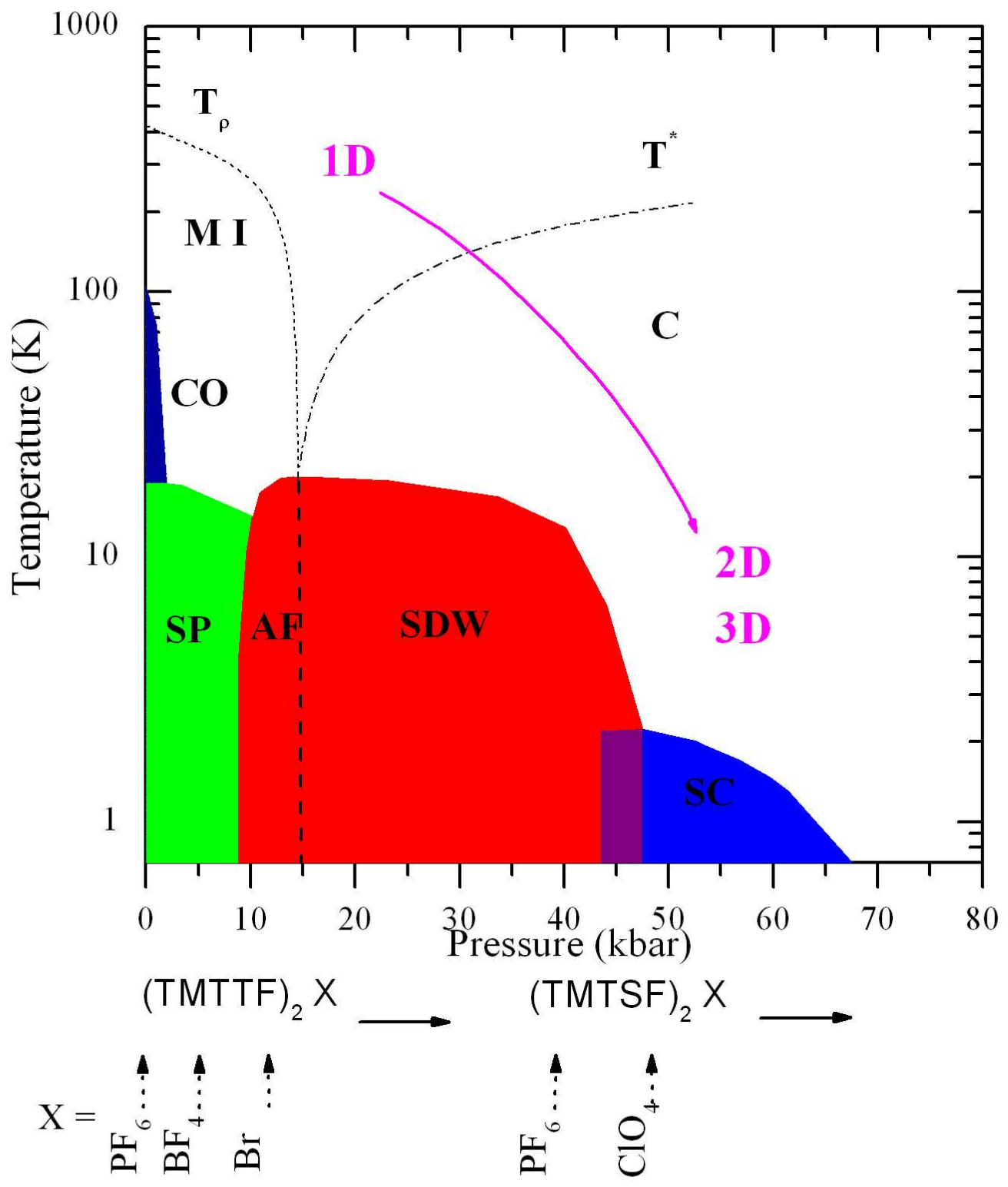

Figure 8: Generic phase diagram for $(\mathrm{TM})_{2} \mathrm{X}$ compounds[42] based on the sulfur compound $(\mathrm{TMTTF})_{2} \mathrm{PF}_{6}$ for the ambient pressure value. Phases with long range order are shaded. $T_{\rho}$ and $T^{\star}$ mark the onset of $1 \mathrm{D}$ charge localization and $1 \mathrm{D} / 2 \mathrm{D}$ deconfinement respectively. 
can be considered at first glance as protoptypes for 1D physics[45]. What $1 \mathrm{D}$ physics means in a nutshell, is the replacement of Landau-Fermi quasiparticles low lying excitations by decoupled spin and charge collective modes [46, 47]. The model for 1D conductors starting from a linearized energy spectrum for excitations close to the Fermi level and adding the relevant Coulomb repulsions which are responsible for electron scattering with momentum transfer 0 and $2 k_{F}$ is known as the popular Tomonaga-Luttinger (TL) model. In this model the spatial variation of all correlation functions (spin susceptibility at $2 k_{F}$ or $4 k_{F}$, CDW, Superconductivity) exhibit a power law decay at large distance, characterized by a non-universal exponent $K_{\rho}$ (which is a function of the microscopic coupling constants) [48]. However an important peculiarity of $(\mathrm{TM})_{2} \mathrm{X}$ materials make them different from the usual picture of TL conductors. Unlike two-stacks TTF - TCNQ materials, $(\mathrm{TM})_{2} \mathrm{X}$ conductors exhibit a band filing which is commensurate with the underlying $1 \mathrm{D}$ lattice due to the 2:1 stoichiometry imposing half a carrier (hole) per TM molecule. Consequently, given a uniform spacing of the molecules along the stacking axis the unit cell contains $1 / 2$ carrier, i.e. the conduction band is quarter-filled. However, non-uniformity of the intermolecular spacing had been noticed from the early structural studies of (TMTTF) ${ }_{2} \mathrm{X}$ crystals[49] due to the periodicity of the anion packing twice the periodicity of the molecluar packing. This non-uniformity amounts to a dimerization of the intrastack overlap between molecules which is important in the sulfur series (prominent $1 / 2$ Umklapp scattering) but still present although less developed in some members of the (TMTSF $)_{2} \mathrm{X}$ series.

A dramatic consequence of this commensurate situation for $(\mathrm{TM})_{2} \mathrm{X}$ materials is the existence of localization channels due to electron-electron Umklapp scatterings with momentum transfer $4 k_{F}$ (two particles scattering) or $8 k_{F}$ (four particles scattering) corresponding to half or quarter-filled bands respectively[50, 51]. This localization is a typical outcome of $1 \mathrm{D}$ physics in the presence of repulsive interactions and leads to a charge gap $\Delta_{\rho}$ in the quasiparticle spectrum although no ordering is expected at any finite temperature for a purely 1D system. The spin sector remains gap-less on account of the separation between spin and charge degrees of freedom in 1D conductors. Fortunately, most features of the 1D localization and the physical properties of this electron gas can be studied by transport and optical properties of the materials. The most salient effect is the existence of a minimum of resistance at $T_{\rho}$ below which the resistance becomes activated. According to the theory of the commensurate 1D electron gas worked out by a number of authors, using the extended Hubbard, DMRG and bozonisation models, (see reference[52] for further reading) it as been shown that both Umklapp mechanisms compete for the establishment of the charge gap, 1/4 and 1/2 Umpklapp scatterings leading to charge ordered and Mott insulators respectively[53]. The experiment is unable to discriminate which of the two localizing channels is the dominant one at low temperature. In spite of the charge gap a metal-like behaviour of the longitudinal resistance can still be oberved at $T$ larger than $\Delta_{\rho}$ leading to a resistance displaying a power law $\rho(T) \approx T^{\theta}(\theta>0)$. Experimental studies have revealed such a metallic behaviour for the resistance either in (TMTTF) ${ }_{2} \mathrm{X}$ under pressure $[54,55]$ or in (TMTSF $)_{2} \mathrm{X}$ even at ambient pressure[30]. What has been found is a 
sublinear exponent, namely $\alpha=0.93$ for the constant volume temperature dependence of the (TMTSF $)_{2} \mathrm{PF}_{6}$ resistance[54, 55]. Similarly, the optical conductivity reveals a power law frequency dependence at high frequency above a charge gap, $\sigma(\omega) \approx \omega^{-1.3}[56]$. Transport and optical data experiments can both be explained within the theoretical framework of commensurate conductors with commensurability one or two. However, in the case of half-filling (commensurability one) the Luttinger parameter $K_{\rho}$ should be very close to unity (very weak repulsive interactions) since $\theta=4 K_{\rho}-3$. A situation which implies weak coupling $K_{\rho} \approx 1$ would be hard to reconcile with the exchange enhancement of the spin susceptibility[57]. On the other hand, when quarter-filled Umklapp scatterings prevail at high temperature $\theta=16 K_{\rho}-3$ and consequently $K_{\rho}=0.23$ according to the data of $(\mathrm{TMTSF})_{2} \mathrm{PF}_{6}$.

Furthermore, the temperature dependence of the transverse conduction along the least conducting direction provides additional arguments in favour of a predominance of the quarter-filled Umklapp above $T_{\rho}$. The behavior of the (TMTSF) $)_{2} \mathrm{PF}_{6}$ resistance along the $c$ direction displays an insulating behaviour below room temperature going through a maximum around $T^{\star} \approx 80-100 \mathrm{~K}$ and becomes metallic at lower temperatures, although remaining several orders of magnitude above the Mott-Ioffe critical value which is considered as the limit between metal and insulating-like transport [58]. Transverse transport requires the tunneling of Fermions (at variance with the longitudinal transport which is related to $1 \mathrm{D}$ collective modes) between neighbouring chains and therefore probes the amount of quasi particles (QP) existing close to Fermi level. The insulating character of the transverse transport at high temperature can thus be interpreted as the signature of a non Fermi-Landau behavior [59]. When transport along the $c$-direction is incoherent, transverse conductivity probes the physics of the $a-b$ planes namely, the physics of the weakly interacting Luttinger chains in the $a-b$ planes. The resistivity along the least conducting direction depends on the one-electron spectral function of a single chain and reads $\rho_{c}(T) \approx T^{1-2 \alpha}[60]$ where $\alpha$ is related to $K_{\rho},\left(\alpha=\frac{1}{4}\left(K_{\rho}+1 / K_{\rho}-2\right)\right.$.

The temperature $T^{\star}$ at which the $c$-axis transport switches from an insulating to a metallic temperature dependence corresponds to a cross-over between two regimes; a high temperature regime with no QP weight at Fermi energy (possibly a TL liquid in the 1D case) and another regime in which the QP weight increases with decreasing temperature. This picture does not necessarily imply that the transport along the $c$-direction must also become coherent below the cross-over since the $c$-axis transport may remain incoherent with a progressive establishment of a Fermi liquid in $a-b$ planes at temperatures below $T^{\star}$. Consequently, the temperature dependence of transport properties along $a$ and $c$-axes in the $1 \mathrm{D}$ regime above $T^{\star}$ lead to a determination of $K_{\rho}$ of order 0.23 in $(\mathrm{TMTSF})_{2} \mathrm{PF}_{6}$ and $(\mathrm{TMTTF})_{2} \mathrm{PF}_{6}$ under a pressure of $12-15$ kbar respectively. The Luttinger parameter is much smaller in (TMTTF $)_{2} \mathrm{PF}_{6}$ at ambient pressure[54], $\left(K_{\rho}=0.18\right)$ due to the enhancement of correlations in the left region of the phase diagram, fig.8. Optical data measured throughout the entire generic diagram reach fairly similar conclusions[61].

The decrease of the charge gap under pressure,fig ( 8 is due to a weakening of intra chain 
correlations moving from left to right in the diagram. Carriers are confined on the 1D chains and the transverse coupling renormalized by intrachain interactions is not pertinent [62]. However, when this gap becomes of the order of the bare kinetic transverse coupling a close interplay between this gap and the transverse coupling occurs giving rise to the sharp suppression of the localization observed around $15 \mathrm{kbar}$ in $(\mathrm{TMTTF})_{2} \mathrm{PF}_{6}$. At higher pressures the transverse coupling becomes pertinent and approaches the bare coupling increasing smoothly under pressure. The deconfinement of the carriers is observed around $T^{\star}$. Below this deconfinement temperature charge excitations lose their 1D character and resemble more and more what is expected in Fermi liquids (quasiparticles), leading in turn to a quadratic temperature dependence for the longitudinal resistivity. However, electron excitations of this "Fermi liquid" retain a low energy gap in the far infra-red spectrum in which most of the oscillator strength is carried by states above the gap coexisting with a very narrow and intense zero frequency peak in the conductivity $[63,56]$. A phase transition towards a long range ordered insulating phase has been observed recently in the charge localized temperature domain. The phase at low temperature has been ascribed according to NMR data to the onset of a charge disproportionation between molecules on the molecular chains[64]. Since the charge of this low temperature phase (see, the CO state on the left side of the generic (TMTTF $)_{2} \mathrm{PF}_{6}$ diagram, figure (8), is no longer uniform some ferroelectricity can be expected as shown by a signature in dielectric measurements [65] . The stability of the CO state (often called a Wigner state) is a direct consequence of the long range nature of the Coulomb repulsion which, in terms of the extended Hubbard model, amounts to finite on-site $\mathrm{U}$ and second-neighbours $\mathrm{V}$ repulsions.

\section{$5 \quad$ Nature of organic superconductivity}

From the very beginning of organic superconductivity, the finding of a common border between an insulating SDW phase and the SC state[30] and even of a coexistence pressure domain for both instabilities[66] raised a great and controversial interest in the nature of the SC pairing. While NMR studies in the early days concluded in favour of nodes in the SC gap[68], thermal conductivity data supported the model of a $s$-type BCS gap covering the entire Fermi surface[69]. Regarding the spin part of the SC wave function, a triplet pairing was claimed from a divergence of the critical field $H_{c 2}(T)$ exceeding the Pauli limiting value reported at low temperature in $(\mathrm{TMTSF})_{2} \mathrm{PF}_{6}$ under pressure when $H$ is applied along the $b^{\prime}$ or $a$ axes [70] and from the absence of a change in the ${ }^{77} \mathrm{Se}$ Knight shift at $T_{c}$ [71]. However, the recent finding of a $T_{c}$ determined by the value of the electron lifetime in the presence of non magnetic lattice defects has conclusively ruled out the s-wave hypothesis and suggested the existence of nodes with zeros of the SC gap on the Fermi surface[67]. This latter experiment performed in (TMTSF) ${ }_{2} \mathrm{ClO}_{4}$ could still not discriminate between the options singlet-d or triplet-p (f)[73]. However, the clear change of the ${ }^{77} \mathrm{Se}$ Knight shift observed at $T_{c}$ in the compound (TMTSF) ${ }_{2} \mathrm{ClO}_{4}[72]$ at fields lower 


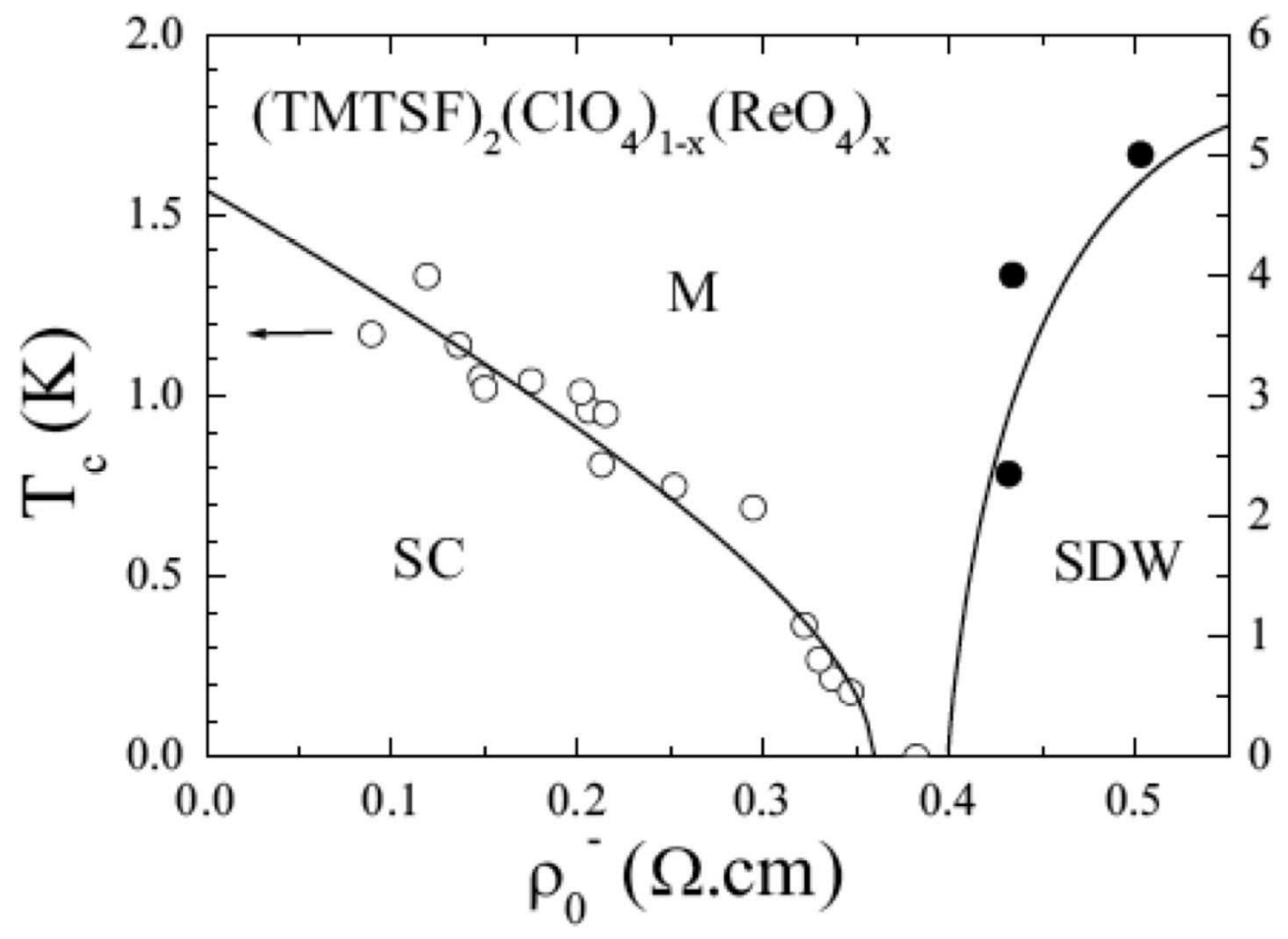

Figure 9: Phase diagram of $(T M T S F)_{2}\left(\mathrm{ClO}_{4}\right)_{(1-x)}\left(\mathrm{ReO}_{4}\right)_{x}$ governed by non magnetic disorder acording to reference[67]. All circles (open and close) refer to very slowly cooled samples in the R-state with different $\mathrm{ReO}_{4}^{-}$contents. 


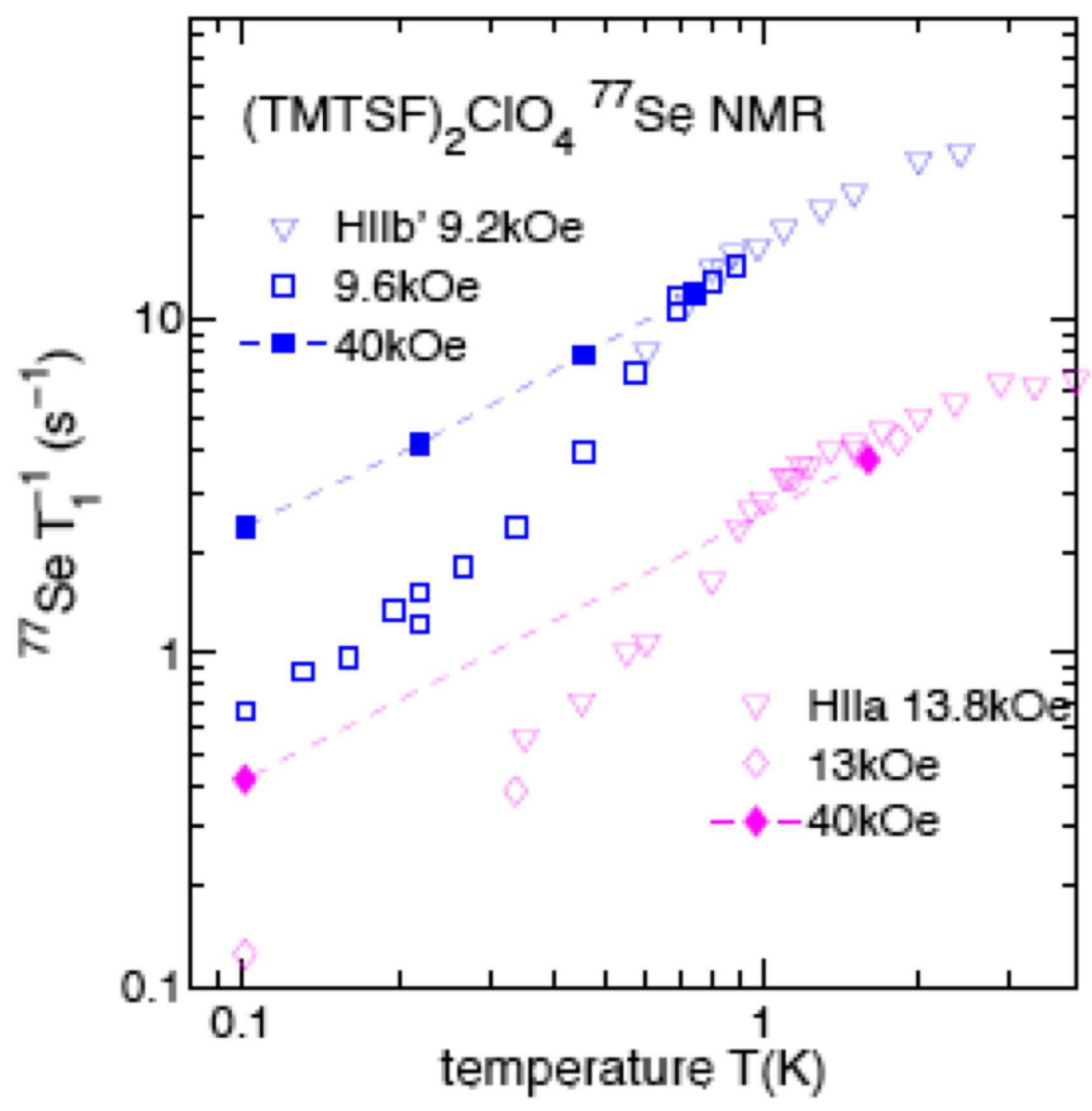

Figure 10: ${ }^{77} \mathrm{Se} T_{1}^{-1}$ vs $\mathrm{T}$ for (TMTSF) ${ }_{2} \mathrm{ClO}_{4}$, for $H / / b^{\prime}$ and $a$, according to reference [72]. 
than those used in the previous (TMTSF) ${ }_{2} \mathrm{PF}_{6}$ studies[71] provided a conclusive evidence in favour of singlet pairing. In addition, a sudden increase of the spin lattice relaxation rate for both field orientations $\| a$ and $b^{\prime}$ provided some evidence for a sharp cross-over or even a phase transition occuring at low temperature under magnetic field between the low field d-wave singlet phase and a high field regime being either a triplet-paired state[74, 75] or an inhomogenous Fulde-Ferrell-Larkin-Ovchinnikov state[76, 77]. A very recent study of the anisotropic superconductiity of (TMTSF $)_{2} \mathrm{ClO}_{4}$ with a magnetic field lying in the $b^{\prime}-a$ plane has shown the survival of superconductivity far above $H_{P}$ and suggested the existence of modulated superconducting phases when high fields are nearly aligned along this cristallographic plane[78, 79] with possibly different kinds of inhomogenous phases for the $a$ and $b^{\prime}$ directions. As far as the origin of the coupling is concerned the incipient antiferromagnetism of these $(\mathrm{TM})_{2} \mathrm{X}$ superconductors supports the existence of strong intrachain repulsive interactions making in turn the usual phonon mediated pairing unlikely to be active. In order to explain the stability of superconductivity once the SDW state is suppressed by a large enough departure from good nesting of the Fermi surface, both instabilities, Peierls (electron-hole) and pairing (electron-electron) have to be treated on equal footings and in particular the ladder diagrammatic summation inherent to the BCS treatment can no longer be used. The most salient outcome of such a treatment is an attraction between carriers on neighbouring chains mediated by the interchain exchange of AF intrachain spin fluctuations [80] provided by the existence of a finite transverse coupling $t_{\perp}$, see figure 11 .

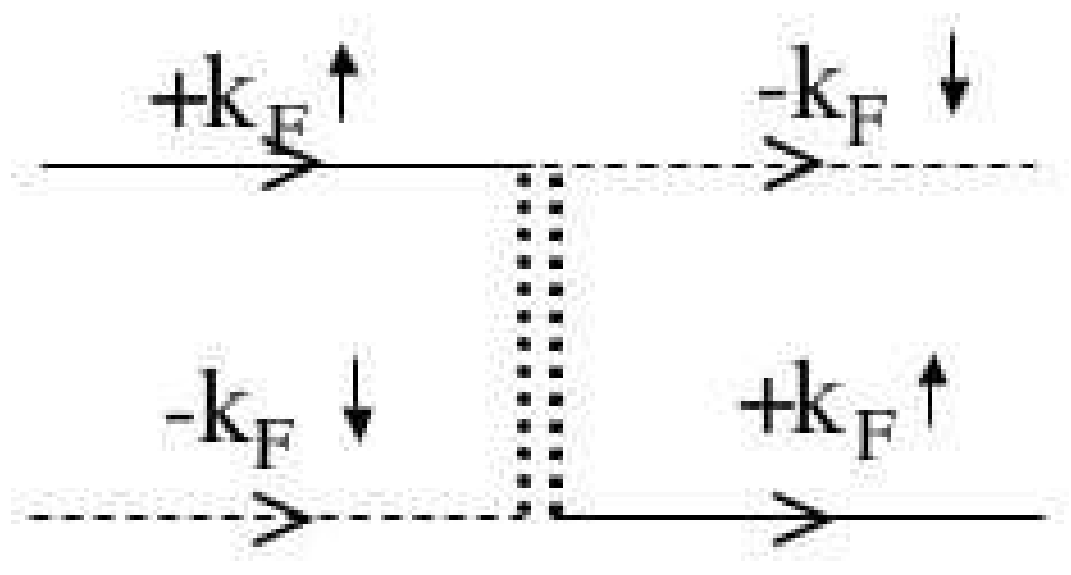

Figure 11: Diagrammatic representation of the interchain attractive pairing according to reference [80].Carriers forming singlet pairs belong to neigbouring chains and exchange an antiferromagnetic spin fluctuation with momentum $2 k_{F}$, the double-dotted line in the scattering process. 


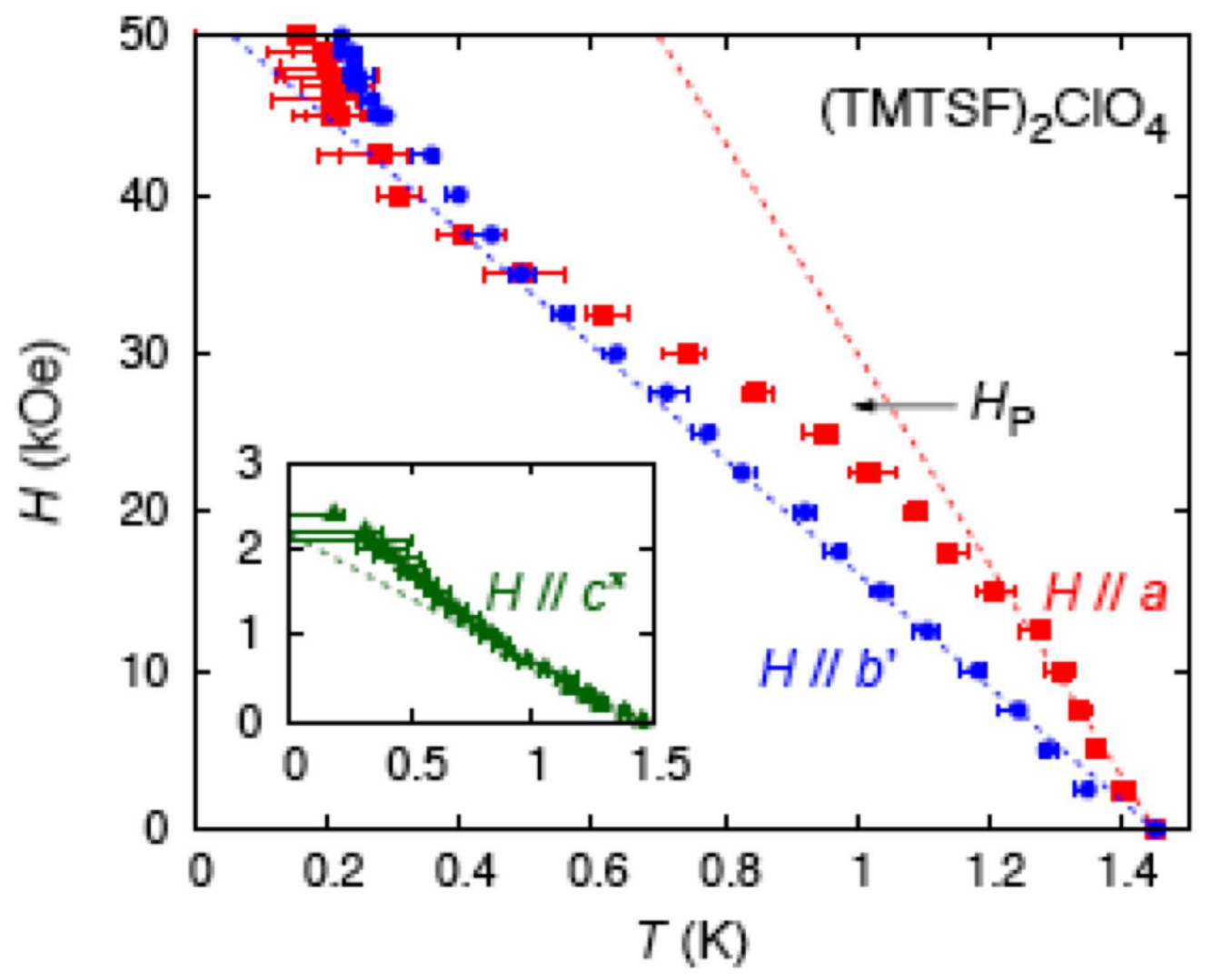

Figure 12: Magnetic fields v.s. $T_{c}$ of (TMTSF) ${ }_{2} \mathrm{ClO}_{4}$ for $H / / a$ (filled squares) and $H / / b^{\prime}$ (filled circles) according to reference[78]. The arrow indicates the estimated value of $H_{P}=26.7 \mathrm{kOe}$.

\section{Conclusion}

This short presentation as shown how research on organic superconductors has developed and has been boosted by two stimulating proposals: first, the theoretical model suggested by Little for high $T_{c}$ organic superconductivity and second, the claims made by the Penn group for precursor signs of superconductivity in the first organic conductors TTF - TCNQ. Whereas both statements were probably overemphasized, the discovery of organic superconductivity in 1980 has shown how interesting this class of materials can be in terms of the low dimensional physics. In addition, the determining role of high pressure governing their physical properties, 1D Mott or Wigner localization and the profusion of 
various instabilities at low temperature have shown how organic conductors compare fairly well in terms of scientific interest with the celebrated class of high $T_{c}$ cuprates. A short article like this one has not been able to survey other important aspects of the physics of 1D conductors such as the remarkable role played by the magnetic field either on the angular magnetoresistance in the normal phase, (see[81] for a recent survey) or in the stabilisation of insulating spin density wave phases with a quantization of the Hall effect[82, 83, 84, 85, 86]. While the actual pairing mechanism behind organic superconductivity is still an enigma this whole field remains particularly challenging, even thirty years after its discovery.

The search, discovery and study of organic superconductors has been a major research domain for the Solid State Physics Laboratory at Orsay over the last thirty years. This period has been characerized by an intense and fruitful cooperation between theoreticians and experimentalists. I wish to address my sincere and deepest thanks to all our co-workers.

\section{References}

[1] H.K. Onnes. Proc. Akad. Wetenshaffen, 14:113, 1911.

[2] J. Bardeen, L.N. Cooper, and J.R. Schrieffer. Phys. Rev., 108:1175, 1957.

[3] H. Fröhlich. Proc.Roy.Soc.A, 223:296, 1954.

[4] G.F. Hardy and J.K. Hulm. Phys.Rev., 93:1004, 1954.

[5] J.Labbé and J.Friedel. J.Physique, 27:153, 1966.

[6] M.Weger and I.R.Goldberg. in Solid State Physics, volume 28. Academic Press, New York, 1973.

[7] J.Labbé, S.Barisić, and J.Friedel. Phys.Rev.Lett, 19:1039, 1967.

[8] J Hulm and B. Matthias. Science, 208:881, 1980.

[9] A. B. Migdal. Sov.Phys.JETP., 7:996, 1958.

[10] G. M. Eliashberg. Sov.Phys.JETP., 11:696, 1960.

[11] V.L.Ginzburg. Jour.Experimental.Theoretical.Physics.(JETP), 47:2318, 1964.

[12] V.L.Ginzburg. Phys.Lett., 11:101, 1964.

[13] W.A. Little. Phys. Rev. A, 134:1416, 1964.

[14] W.A. Little. Scientific American, 212:21, 1965.

[15] H.N. McCoy and W.C. Moore. J. Am. Chem. Soc., 33:273, 1911. 
[16] F. London. Jour of Chemical Physics, 5:837, 1937.

[17] H. Akamatsu, H. Inokuchi, and Y. Matsunaga. Nature, 173:168, 1954.

[18] W.A. Little. J. Polymer. Sci C, 29:17, 1970.

[19] R.E. Peierls. in Quantum Theory of Solids. Oxford University Press, London, 1955.

[20] R. Comès, M. Lambert, H. Launois, and H. R. Zeller. Phys. Rev. B, 8:571, 1973.

[21] Yu. A Bychkov, L. P. Gorkov, and I.E. Dzyaloshinskii. Sov. Phys. JETP, 23:489, 1966.

[22] F. Wudl, G.M. Smith, and E.J. Hufnagel. J. Chem. Soc. Chem. Comm, page 1453, 1970.

[23] R. Comés. In H. J. Keller, editor, Chemistry and Physics of One-Dimensional Metals, page 315. Plenum Press, New York, 1977.

[24] J. Ferraris, D.O. Cowan, W. Walatka, and J.H. Perlstein. J. Am. Chem. Soc, 95:948, 1973.

[25] L.B. Coleman, M.J. Cohen, D.J. Sandman, F.G. Yamagishi, A.F. Garito, and A.J. Heeger. Solid State Comm, 12:1125, 1973.

[26] G.A.Thomas et-al. Phys.Rev., B13:5105, 1976.

[27] Z.Z. Wang, J.C. Girard, C. Pasquier, D. Jérome, and K. Bechgaard. Phys. Rev. B., 67:R-121401, 2003.

[28] F. Denoyer, R. Comès, A.F. Garito, and A.H. Heeger. Phys. Rev. Lett., 35:445, 1975.

[29] S. Megtert, R. Comès, C. Vettier, R. Pynn, and A. F. Garito. Solid State Commun., 31:977, 1979.

[30] D. Jérome and H.J. Schulz. Adv in Physics, 31:299, 1982.

[31] R. H. Friend, M. Miljak, and D. Jérome. Phys. Rev. Lett., 40:1048, 1978.

[32] J. P. Pouget, S. K. Khanna, F. Denoyer, R. Comès, A. F. Garito, and A. J. Heeger. Phys. Rev. Lett., 37:437, 1976.

[33] S. Yasuzuka, K. Murata, T. Arimoto, and R. Kato. Jour. Phys. Soc. Japan, 76:033701, 2007.

[34] B. Horovitz, H. Gutfreund, and M. Weger. Phys. Rev. B, 12:3174, 1975.

[35] A.F. Garito and A.J. Heeger. Accounts of Chemical Research, 7:232, 1974. 
[36] E.M. Engler, B.A. Scott, S. Etemad, T. Penney, and V.V. Patel. Jour. Am. Chem. Soc., 99:5909, 1977.

[37] J.R. Andersen, K. Bechgaard, C.S. Jacobsen, G. Rindorf, H. Soling, and N. Thorup. Acta Cryst.B., 34:1901, 1978.

[38] A. Andrieux, P.M. Chaikin, C. Duroure, D. Jérome, C. Weyl, K. Bechgaard, and J.R. Andersen. J. Physique. Paris, 40:1199, 1979.

[39] J.L. Galigne, B. Liautard, S. Peytavin, G. Brun, , J.-M. Fabre, E. Torreilles, and L. Giral. Acta Cryst., B 34:620, 1978.

[40] K. Bechgaard, C.S. Jacobsen, K. Mortensen, H.J. Pedersen, and N. Thorup. Solid.State.Comm, 33:1119, 1979.

[41] D. Jérome, A. Mazaud, M. Ribault, and K. Bechgaard. J. Phys. (Paris) Lett., 41:L95, 1980.

[42] D. Jérome. Science, 252:1509, 1991.

[43] H. Wilhelm, D. Jaccard, R. Duprat, C. Bourbonnais, D. Jérome, J. Moser, C. Carcel, and J.M. Fabre. Eur. Phys. Jour., B,21:175, 2001.

[44] T. Adachi, E. Ojima, K. Kato, and H. Kobayashi. Jour. Am. Chem. Soc., 122:3238, 2000 .

[45] C. Bourbonnais and D. Jérome. Science, 281:1156, 1998.

[46] H. J. Schulz. Phys. Rev. Lett., 64:2831, 1990.

[47] J. Voit. Rep. Prog. Phys., 58:977, 1995.

[48] J. Solyom. Adv. Phys., 28:201, 1979.

[49] L. Ducasse, A. Abderraba, J. Hoarau, M. Pesquer, B. Gallois, and J. Gaultier. J. Phys. C, 39:3805, 1986.

[50] V. J. Emery, R. Bruinsma, and S. Barisić. Phys. Rev. Lett., 48:1039, 1982.

[51] T. Giamarchi. Physica, B230-232:975, 1997.

[52] T. Giamarchi. Quantum Physics in One-Dimension. Clarendon Press, Oxford, 2004.

[53] M. Tsuchiizu, H. Yoshika, and Y. Suzumura. Jour. Phys. Soc. Japan, 70:1460, 2001.

[54] P. Auban, D. Jérome, C. Carcel, and J.M. Fabre. J.Phys.IV France, 114:41, 2004. and to be published. 
[55] L. Degiorgi and D. Jérome. Jour. Phys. Soc. Japan, 75:051004, 2006.

[56] A. Schwartz, M. Dressel, G. Grüner, V. Vescoli, L. Degiorgi, and T. Giamarchi. Phys. Rev. B., 58:1261, 1998.

[57] C.Bourbonnais and D.Jérome. Advances in synthetic metals. pages 206-261. Elsevier, New York, 1999.

[58] N.F. Mott. Metal-Insulator Transitions. Taylor and Francis, London, 1974.

[59] J. Moser, M. Gabay, P. Auban-Senzier, D. Jérome, K. Bechgaard, and J. M. Fabre. Eur. Phys. Jour. B, 1:39, 1998.

[60] A. Georges, T. Giamarchi, and N. Sandler. Phys. Rev. B., 61:16393, 2000.

[61] A. Pashkin, M. Dressel, and C. A. Kuntscher. Phys. Rev.B., 74:165118, 2006.

[62] C. Bourbonnais, F. Creuzet, D. Jérome, K. Bechgaard, and A. Moradpour. J. Phys. (Paris) Lett., 45:L755, 1984.

[63] N. Cao T. Timusk and K. Bechgaard. J. Physique (France), 6:1719, 1996.

[64] D.S. Chow, F. Zamborsky, B. Alavi, D.J. Tantillo, A. Baur, C.A. Merlić, and S.E. Brown. Phys. Rev. Lett., 85:1698, 2000.

[65] F. Nad, P. Monceau, C. Carcel, and J.M. Fabre. Phys. Rev. B., 62:1753, 2000.

[66] T. Vuletić, P. Auban-Senzier, C. Pasquier, S. Tomić, D. Jérome, M. Héritier, and K. Bechgaard. Eur. Phys. J. B, 25:319, 2002.

[67] N.Joo, P. Auban-Senzier, C. Pasquier, D.Jérome, and K. Bechgaard. Eur.Phys.Lett, 2005.

[68] M. Takigawa and G. Saito. J. Phys. Soc. Jpn, 55:1233, 1986.

[69] S. Belin and K. Behnia. Phys. Rev. Lett., 79:2125, 1997.

[70] I. J. Lee, M. J. Naughton, G. M. Danner, and P. M. Chaikin. Phys. Rev. Lett., 78:3555, 1997.

[71] I. J. Lee, S. E. Brown, W. G. Clark, M. J. Strouse, M. J. Naughton, W. Kang, and P.M. Chaikin. Phys.Rev.Lett., 88:017004, 2001.

[72] J. Shinagawa, Y. Kurosaki, F. Zhang, C. Parker, S. E. Brown, and D. Jérome. Phys.Rev.Lett., 98:147002, 2007.

[73] J. C. Nickel, R. Duprat, C. Bourbonnais, and N. Dupuis. Phys. Rev. Lett., 95:247001, 2005. 
[74] H. Shimahara. Jour. Phys. Soc. Japan, 69:1966, 2000.

[75] N. Belmechri, G. Abramovici, M. Héritier, S. Haddad, and S. Charfi-Kaddour. Eur. Phys. Lett, 80:37004, 2007.

[76] P. Fulde and R. A. Ferrell. Phys. Rev., 135:A550, 1964.

[77] A. I. Larkin and Y. N. Ovchinnikov. Sov. Phys. JETP., 20:762, 1965.

[78] S. Yonezawa, Y. Maeno, P. Auban-Senzier, C. Pasquier, K. Bechgaard, and D. Jérome. Phys. Rev. Lett. submitted.

[79] S. Yonezawa, Y. Maeno, P. Auban-Senzier, C. Pasquier, and D. Jérome. J. Phys. Soc. Japan. submitted.

[80] L. G. Caron and C. Bourbonnais. Physica, 143B:453, 1986.

[81] T. Osada and E. Ohmichi. Jour. Phys. Soc. Japan, 75:051006, 2006.

[82] M. Ribault, D. Jérome, J. Tuchendler, C. Weyl, and K. Bechgaard. J. Phys. Lett., 44:L-953, 1983.

[83] L. P. Gorkov and A. G. Lebed. J. Phys. (Paris) Lett., 45:L433, 1984.

[84] M. Héritier, G. Montambaux, , and P. Lederer. J. Phys. (Paris) Lett., 45:L943, 1984.

[85] J.R. Cooper, W. Kang, P. Auban, G. Montambaux, D. Jérome, and K. Bechgaard. Phys. Rev. Lett, 65:1984, 1989.

[86] S.T. Hannahs, J.S. Brooks, W. Kang, L.Y. Chiang, and P.M. Chaikin. Phys.Rev.Lett., 63:1988, 1989. 